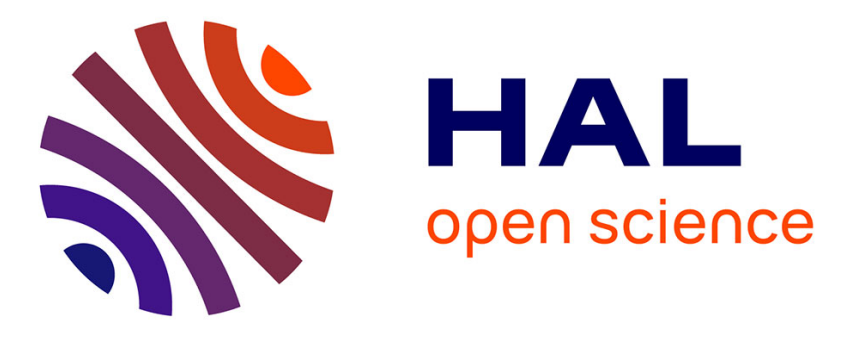

\title{
Acoustic phonon sideband dynamics during polaron formation in a single quantum dot
}

Daniel Wigger, Vage Karakhanyan, Christian Schneider, Martin Kamp, Sven Höfling, Pawel Machnikowski, Tilmann Kuhn, Jacek Kasprzak

\section{- To cite this version:}

Daniel Wigger, Vage Karakhanyan, Christian Schneider, Martin Kamp, Sven Höfling, et al.. Acoustic phonon sideband dynamics during polaron formation in a single quantum dot. Optics Letters, 2020, 45 (4), pp.919. 10.1364/OL.385602 . hal-02995750

\section{HAL Id: hal-02995750 \\ https://hal.science/hal-02995750}

Submitted on 9 Nov 2020

HAL is a multi-disciplinary open access archive for the deposit and dissemination of scientific research documents, whether they are published or not. The documents may come from teaching and research institutions in France or abroad, or from public or private research centers.
L'archive ouverte pluridisciplinaire HAL, est destinée au dépôt et à la diffusion de documents scientifiques de niveau recherche, publiés ou non, émanant des établissements d'enseignement et de recherche français ou étrangers, des laboratoires publics ou privés. 


\title{
Acoustic Phonon Sideband Dynamics During Polaron Formation in a Single Quantum Dot
}

\author{
Daniel Wigger ${ }^{1,6}$, Vage Karakhanyan ${ }^{2}$, Christian Schneider ${ }^{3}$, Martin Kamp ${ }^{3}$,

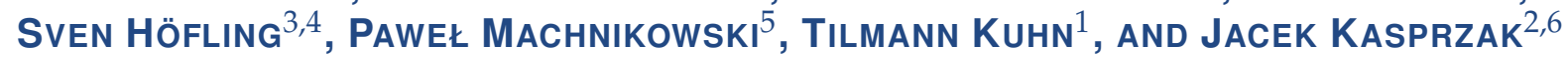 \\ ${ }^{1}$ Institute of Solid State Theory, University of Münster, Wilhelm-Klemm-Str. 10, 48149 Münster, Germany \\ ${ }^{2}$ Universit'e Grenoble Alpes, CNRS, Grenoble INP, Institut Néel, 38000 Grenoble, France \\ ${ }^{3}$ Technische Physik, Universität Würzburg, 97074 Würzburg, Germany \\ ${ }^{4}$ SUPA, School of Physics and Astronomy, University of St. Andrews, St. Andrews KY16 9SS, UK \\ ${ }^{5}$ Department of Theoretical Physics, Wrocław University of Science and Technology, 50-370 Wrocław, Poland \\ ${ }^{6}$ e-mail: d.wigger@wwu.de, jacek.kasprzak@neel.cnrs.fr
}

Compiled January 10, 2020

\begin{abstract}
When an electron-hole pair is optically excited in a semiconductor quantum dot the host crystal lattice needs to adapt to the presence of the generated charge distribution. Therefore the coupled exciton-phonon system has to establish a new equilibrium, which is reached in the form of a quasiparticle called polaron. Especially, when the exciton is abruptly generated on a timescale faster than the typical lattice dynamics, the lattice displacement cannot follow adiabatically. Consequently, a rich dynamics on the picosecond timescale of the coupled system is expected. In this study we combine simulations and measurements of the ultrafast, coherent, nonlinear optical response, obtained by four-wave mixing spectroscopy, to resolve the formation of this polaron. By detecting and investigating the phonon sidebands in the four-wave mixing spectra for varying pulse delays and different temperatures we have access to the influence of phonon emission and absorption processes which finally result in the emission of an acoustic wave packet out from the quantum dot. () 2020 Optical Society of America
\end{abstract}

http://dx.doi.org/10.1364/ao.XX.XXXXXX

The coupling between quantum dot (QD) excitons and acoustic phonons is unavoidable in self-assembled systems. On the one hand the dephasing effect of the lattice vibrations is in general seen as detrimental. On the other hand many new approaches focus on the active use of phonons in nanophotonics. Phonon assisted transitions can be used for deterministic state preparation [1] and even information transfer [2,3]. Exciton-phonon coupling can also serve as an interface to induce and optically control the quantum state of localized phonon modes [4] or even the macroscopic motion of mechanical oscillators [5-7].

One important effect associated with this fundamental interaction is that by the generation of an exciton the combined chargephonon system is brought out of equilibrium. By the generation of a localized lattice distortion it reaches a new state of rest. This new eigenstate of the entire system is called a polaron. Due to its fundamental character its investigation has a long history $[8,9]$. When the exciton creation happens significantly faster than the polaron formation the process is accompanied by the emission of a phonon wave packet [10]. Spectrally this phonon assisted type of event appears as characteristic sidebands also in photoluminescence [11-13] and absorption [14] signals in QDs and other single photon emitters $[15,16]$. However, the dynamics of this transition into a new equilibrium has only rarely been studied on single QDs $[17,18]$. Previously, the exciton dephasing on a picosecond time scale due to acoustic phonons was shown on ensembles of QDs $[19,20]$ which, due to the inhomogeneous broadening, does not allow for a spectrally resolved analysis. We here focus on the back-action of the polaron formation onto the excitons' coherent spectra, which are provided by four-wave mixing (FWM) spectroscopy realized with heterodyne spectral interferometry [21]. Applying a two pulse excitation with a variable delay $\tau_{12}$ within the pair of pulses, directly measures the evolution of the microscopic exciton polarization, i.e., its coherence. By this approach we have access to the spectral and time domain at the same time.

A schematic picture of our study is shown in Figs. 1(a)-(d). The investigated system is a single self-assembled (In)GaAs semiconductor QD embedded in a planar semiconductor microcavity $[22,23]$. Its low quality factor of around 160 allows for a spectral matching with $150 \mathrm{fs}$ chirp-corrected laser pulses. Simultaneously, owing to the enhancement of the intra-cavity field, the external resonant excitation can be decreased significantly, resulting in record signal-to-noise ratios for single QD FWM signals. Such an efficient FWM retrieval was a key aspect in current experiments. It enabled detection of phonon sidebands, with field amplitudes more than an order of magnitude below that of the zero phonon line transition.

Prior to optical excitation the QD is in its ground state. The QD is embedded into the host crystal and is distinguished from it by a slightly different lattice constant. However, these details of the atomic structure are not important for this study, therefore we simply mark a part of the schematic crystal lattice in Fig. 1(a) as region of the QD by the green dashed circle. 


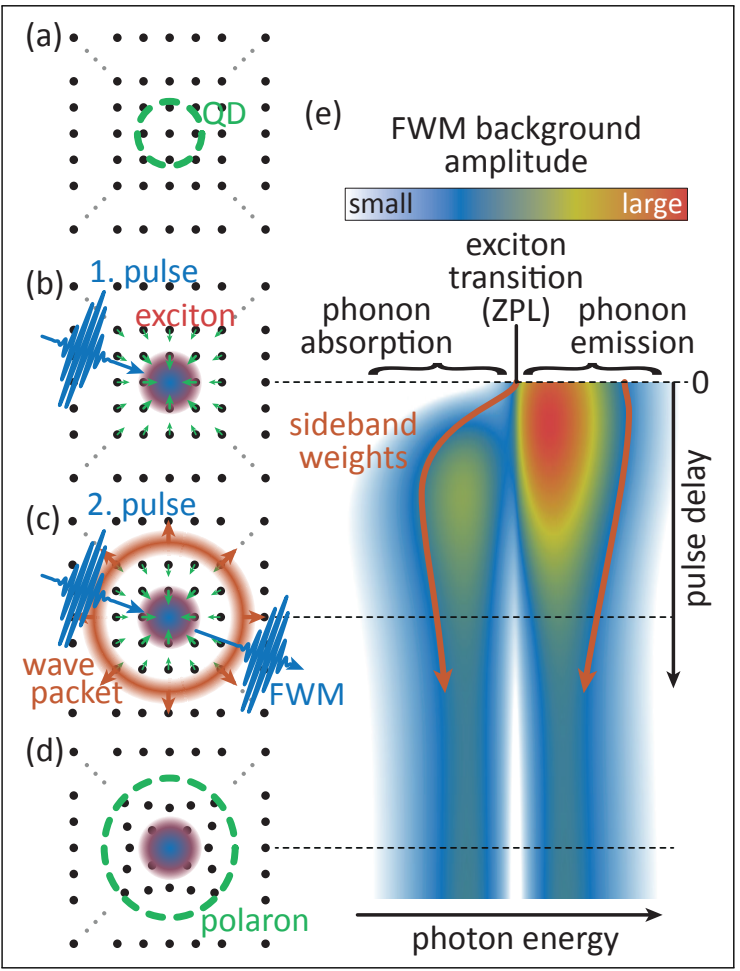

Fig. 1. Schematic picture of the polaron generation and its measurement. (a) Empty QD system in equilibrium. (b) The laser pulse creates an exciton in the QD. The charge distributions attracts the surrounding lattice atoms. (c) The fast contraction of the lattice leads to the emission of a phonon wave packet. The second laser pulse is used to probe the exciton dynamics. (d) New equilibrium state in the presence of an exciton is called polaron. (e) Phonon background spectrum of the FWM signal. The delay increases from top to bottom. The dashed lines loosely correspond to the schematics in (b), (c), and(d). The orange arrows follow the intensity distribution of the two sidebands.

The first exciting laser pulse resonantly creates an excitonic polarization in the QD as depicted in Fig. 1(b). This excitation creates a charge distribution which is felt by the surrounding atoms making them move towards the QD center. When the exciton generation happens on a timescale faster than the typical timescale of the phonons, i.e. approximately below 1 picosecond, it is accompanied by the emission of a phonon wave packet as sketched in Fig. 1(c) [10]. In the final eigenstate, the coupled exciton-phonon system forms a polaron consisting of the exciton in the QD and a distorted lattice in the region of the dot. This is illustrated in Fig. 1(d). To probe the back-action of the polaron formation onto the exciton, after a delay time $\tau_{12}$ the system is excited by a second laser pulse. The arrival time of this second pulse is varied to measure the exciton coherence dynamics. As depicted in (c), the second pulse creates the FWM signal carrying information on the microscopic polarization of the exciton.

Assuming a pure-dephasing type coupling to acoustic phonons, the FWM signal can be obtained analytically in the limit of ultrafast laser pulses treated as delta functions. For this purpose, the optical excitation with two pulses carrying phases $\varphi_{1}$ and $\varphi_{2}$ of the excitonic two level system is calculated. As shown in Ref. [24], the microscopic polarization of the exciton filtered with respect to the FWM phase $\varphi_{\mathrm{FWM}}=2 \varphi_{2}-\varphi_{1}$ reads

$$
\begin{aligned}
& p_{\mathrm{FWM}}\left(t, \tau_{12}\right) \sim \exp \left\{\sum _ { \mathbf { q } } | \frac { g _ { \mathbf { q } } } { \omega _ { q } } | ^ { 2 } \left[2 \cos \left(\omega_{q} t\right)-3\right.\right. \\
& \left.\left.+e^{i \omega_{q} \tau_{12}}\left(2-e^{i \omega_{q} t}\right)-n_{q}\left|e^{i \omega_{q} \tau_{12}}\left(2-e^{i \omega_{q} t}\right)-1\right|^{2}\right]\right\},
\end{aligned}
$$

with $t, \tau_{12}>0$. Here, a frame that rotates with the exciton's transition energy was chosen. The exciton-phonon coupling matrix element for the usual deformation potential coupling reads

$$
g_{\mathbf{q}}=\frac{q}{\sqrt{2 \rho \hbar V \omega_{q}}}\left[D_{\mathrm{e}} e^{-q^{2} a_{\mathrm{e}}^{2} / 4}-D_{\mathrm{h}} e^{-q^{2} a_{\mathrm{h}}^{2} / 4}\right],
$$

where the mass density $\rho$ and the deformation potentials for electrons and holes $D_{\mathrm{e}}$ and $D_{\mathrm{h}}$, respectively, are material parameters. It has been found that such a spherical parametrization with the localization lengths $a_{\mathrm{e}}$ and $a_{\mathrm{h}}$ taken as suitable fit parameters also well reproduces the phonon coupling of QDs with an anisotropic shape [25].

Converting the summation over the phonon wave vectors $\mathbf{q}$ into a spectral integration by the definition of the phonon spectral density $J\left(\omega_{\mathrm{ph}}\right)=\int\left|g_{q}\right|^{2} \delta\left(\omega_{\mathrm{ph}}-\omega_{q}\right) \mathrm{d}^{3} q$ and taking the temperature $T=0$, which leads to a vanishing initial Bose distribution of the phonons $n_{q}=0$ the FWM polarization becomes

$$
\begin{array}{r}
p_{\mathrm{FWM}}\left(t, \tau_{12}\right) \sim \exp \left\{\int \frac { J ( \omega _ { \mathrm { ph } } ) ^ { 2 } } { \omega _ { \mathrm { ph } } ^ { 2 } } \left[e^{i \omega_{\mathrm{ph}} t}+e^{-i \omega_{\mathrm{ph}} t}-3\right.\right. \\
\left.\left.+e^{i \omega_{\mathrm{ph}} \tau_{12}}\left(2-e^{i \omega_{\mathrm{ph}} t}\right)\right] \mathrm{d} \omega_{\mathrm{ph}}\right\} .
\end{array}
$$

The Fourier transform of this polarization with respect to the real time after the second pulse $t$ gives the phonon sidebands of the FWM spectrum for a given delay $\tau_{12}$. For the special case of $\tau_{12}=0$ the dynamics of the FWM polarization reads

$$
p_{\mathrm{FWM}}(t, 0) \sim \exp \left[\int \frac{J\left(\omega_{\mathrm{ph}}\right)^{2}}{\omega_{\mathrm{ph}}^{2}}\left(e^{i \omega_{\mathrm{ph}} t}-1\right) \mathrm{d} \omega_{\mathrm{ph}}\right],
$$

which directly shows that only spectral features at positive energies are expected when the two laser pulses hit the QD at the same time. From Eq. (3) the phonon contribution is isolated by subtracting the constant long time value $p_{\mathrm{FWM}}\left(t \rightarrow \infty, \tau_{12}\right)$. An exemplary delay resolved simulation of this background is shown in Fig. 1(e), where the delay increases from $\tau_{12}=0$ at the top to the bottom. We find that the spectrum for most $\tau_{12}$ consists of two maxima located energetically below and above the zero phonon line (ZPL), i.e., the exciton transition. Because for positive delays the FWM signal is a measure for the coherence in the system the phonon sidebands can be seen as phonon assisted coherences of the system. We focus first on the vanishing delay case and clearly see that the phonon sideband only appears on the high energy side of the ZPL, as already predicted from Eq. (4). Because we consider a vanishing temperature initially no phonons are present $\left(n_{q}=0\right)$ that could be absorbed. Therefore we know that this sideband stems from phonon emission processes. Because the FWM experiment for a vanishing delay is a photon absorption process this phonon sideband appears at the high energy side of the ZPL. It is known that this phonon emission leads to the generation of a wave packet leaving the QD at the speed of sound [26]. Once this acoustic pulse has 
left the region of the QD its phonons cannot be absorbed during optical excitations of the exciton, meaning that they cannot give rise to sidebands in the FWM spectrum. Therefore only the generated polaron leads to the remaining sidebands for large $\tau_{12}$, at the bottom of Fig. 1(e). We find that these sidebands now appear symmetrically on both sides of the ZPL and are much less pronounced than the sidebands at short delays. This can be interpreted as remaining phonon assisted coherence in the polaron state. Following the phonon absorption sideband, energetically below the exciton, back towards short delays we see that also this process becomes more pronounced before it vanishes at $\tau_{12}=0$. From the general dynamical behavior of the phonon sidebands we can conclude that significant variations appear during the polaron generation, i.e., during the phonon wave packet emission. Consequently, while the traveling acoustic pulse is still overlapping with the QD the entire phonon background is increased promoting not only reabsorption processes, but also stimulated phonon emission.

In Refs. [24, 27] the aforesaid behavior of the phonon sidebands was already calculated and additional calculations for increased temperatures predicted a symmetrization of the sidebands with respect to the phonon emission and absorption side of the ZPL. To confirm this prediction we perform the first measurement of the phonon sideband dynamics during the polaron formation process resolved by FWM spectroscopy. Figure 2 shows the measured FWM spectra dynamics in the left column for the temperatures $5 \mathrm{~K}, 20 \mathrm{~K}$, and $30 \mathrm{~K}$, in (a), (b), and (c), respectively. Note that the experiment at $30 \mathrm{~K}$ was performed on a different dot in the same sample, which might lead to some variations of the geometry of the QDs, although, these are estimated to be weak [28], especially due to the sample annealing, which is known to decrease the fluctuations of size and Indium distribution within the ensemble of InGaAs QDs [29]. The strongest signal in these QD systems naturally defines the ZPL and therefore appears at the exciton transition with $E-E_{\mathrm{ZPL}}=0$. The detected phonon sidebands spread over a few $\mathrm{meV}$ and disappear within around 2 ps. The laser pulses used for the excitation of the system have a duration of approximately $150 \mathrm{fs}$. In the detected spectral dynamics we directly see that the polaron formation dynamics happens mainly within approximately 1 picosecond. In this context simulations in the limit of delta-pulses would not be justified because the laser pulses are not sufficiently more rapid with respect to the phonon dynamics. Therefore, to account for finite pulse durations we simulate the optically driven coupled exciton-phonon dynamics in a density matrix approach and use a correlation expansion approach to determine the FWM signal. This approach has been successfully applied before to model the phonon influence on FWM signals [30,31].

The results of the simulations with a pulse duration of $150 \mathrm{fs}$ are plotted in the middle column of Fig. 2 showing a good agreement with the measured data for the three respective temperatures. Both the timescale of the polaron formation, identified by the disappearance of the dominant part of the phonon sidebands (yellow areas), and the energetic spread of the sidebands agree very well. This agreement has been achieved with the localization lengths $a_{\mathrm{e}}=4 \mathrm{~nm}$ and $a_{\mathrm{h}}=0.87 a_{\mathrm{e}}=3.5 \mathrm{~nm}$ and the deformation potentials $D_{\mathrm{e}}=10.5 \mathrm{eV}$ and $D_{\mathrm{h}}=5.25 \mathrm{eV}$. Note that because of the rather similar values of $a_{\mathrm{e}}$ and $a_{\mathrm{h}}$ the phonon coupling is also well reproduced by a standard phonon spectral density with coupling strength $A=0.081 \mathrm{ps}^{2}$ and a cut-off frequency $\omega_{\mathrm{c}}=1.8 \mathrm{ps}^{-1}$ [1]. We want to remark the values of $a_{\mathrm{e} / \mathrm{h}}$ in general do not reflect the real dimensions of the QD but act as a spherical representation leading to the same phonon coupling.
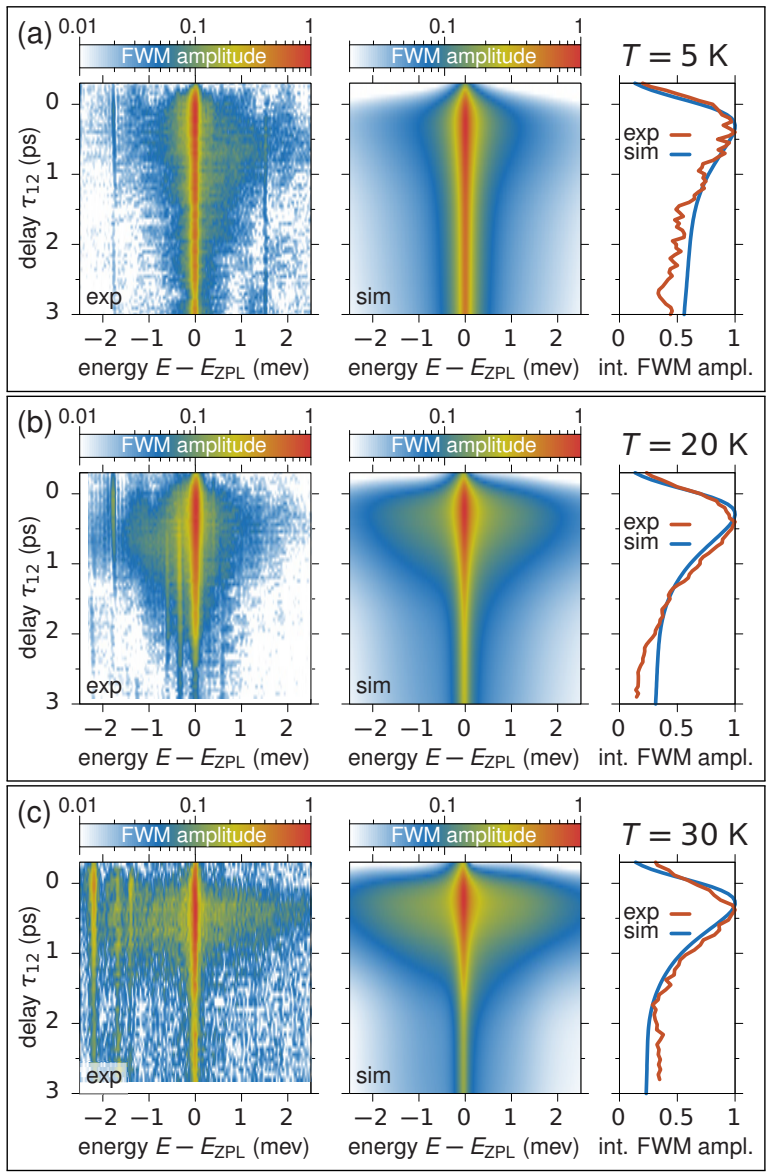

Fig. 2. Measured and simulated FWM spectra for varying pulse delay $\tau_{12}$ left and center, respectively. The corresponding spectrally integrated and normalized FWM amplitudes are shown on the right, experiment in orange and theory in blue. The temperatures are $T=5 \mathrm{~K}$ in (a), $T=20 \mathrm{~K}$ in (b), and $T=30 \mathrm{~K}$ in (c).

As a consequence, also $D_{\mathrm{e}}$ and $D_{\mathrm{h}}$ deviate from the typically used ones by a factor of 1.5 [32]. Nevertheless similar to the discussion in Ref. [17] other choices would not give reasonable agreement, both in the spectral and the time domain. Smaller values for $D_{\mathrm{e} / \mathrm{h}}$ could in principle be compensated by smaller $a_{\mathrm{e} / \mathrm{h}}$, as these would increase the coupling strength. But in turn the spectral spread of the phonon sidebands would increase and the polaron formation time would shorten. Thus the agreement between simulation and experiment would be less good.

Despite the qualitative agreement, it is not straightforward to compare false-color plots quantitatively. To retrieve a quantity that can directly be compared we plot the spectrally integrated FWM amplitude as a function of the delay in the right column. While the rise of the signal around $\tau_{12}=0$ is mainly given by the laser pulse duration, the following decay directly reflects the loss of coherence due to the phonon wave packet emission during the polaron formation. These dynamics are therefore called phonon induced dephasing of the exciton. For all considered temperatures the curves from experiment (orange) and from theory (blue) agree almost perfectly. Therefore we can conclude that the geometry of the investigated QDs are almost equal. As mentioned before, we expect the strongest asymmetry between the phonon emission and absorption sidebands for small 


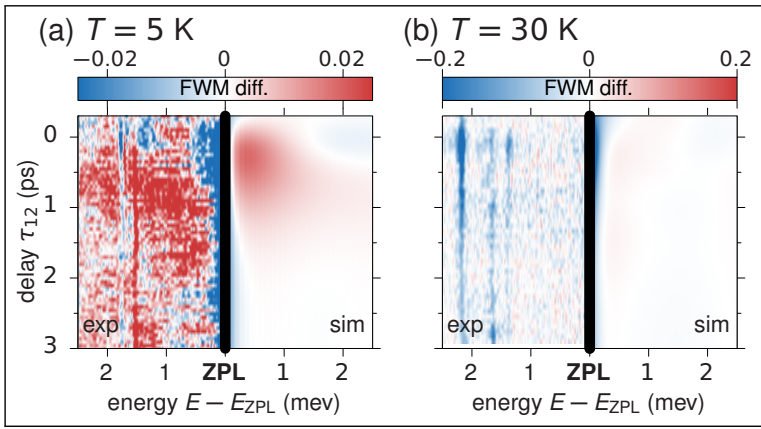

Fig. 3. Difference between phonon emission and phonon absorption side of the FWM spectra for (a) $T=5 \mathrm{~K}$ and (b) $T=30 \mathrm{~K}$. The difference is calculated by subtracting the FWM amplitude for $E-E_{\mathrm{ZPL}}<0$ from the one at $E-E_{\mathrm{ZPL}}>0$. The experiment is shown on the left and the simulation on the right, respectively. To isolate the influence of the phonon sidebands the black line blocks the ZPL influence.

temperatures. For larger temperatures more and more phonon modes are already occupied before the first laser pulse and the ratio between absorption $\sim n_{q}$ and stimulated emission $\sim\left(n_{q}+1\right)$ becomes more balanced. This is what we also find here. When focusing on the $T=5 \mathrm{~K}$ example in Fig. 2(a) we find a stronger sideband at energies above the ZPL for delays around $\tau_{12}=0.5 \mathrm{ps}$. This is in agreement with the $T=0$ example in Fig. 1(e). To confirm this asymmetry in Fig. 3(a) we plot the difference between the phonon emission and the absorption side of the FWM spectrum for $T=5 \mathrm{~K}$. The left side of the plot shows the experiment and the right side the simulation. We find significant positive values in both cases until $\tau_{12}=2 \mathrm{ps}$ predominantly around $\tau_{12}=0.5 \mathrm{ps}$. This confirms that the high energy side of the ZPL has stronger signals then the respective ones below the ZPL. When increasing the temperature via $T=20 \mathrm{~K}$ in Fig. $2(\mathrm{~b})$ to $T=30 \mathrm{~K}$ in (c) the sidebands get more and more symmetric because the contributions stemming from occupied phonon modes $\sim n_{q}$ overweigh the spontaneous processes $\sim 1$. To also confirm this symmetrization Fig. 3(b) shows the difference spectra for $T=30 \mathrm{~K}$. For the entire delay range the measured signal difference shows equal positive and negative values, which proves that the phonon emission and absorption are balanced. This is in good agreement with the simulated signal difference.

In conclusion, we have provided the first demonstration of the polaron formation in a single semiconductor QD detected in FWM spectroscopy. In agreement with simulations this process takes a few picoseconds and manifests in characteristic dynamics of phonon sidebands. Also the temperature of the system influences details of the detected sidebands because the occupation of the contributing phonon modes strongly influences the strength of phonon absorption and emission probabilities.

Our work shows that experimental and theoretical insight into coherent dynamics of phonon assisted processes in nanostructures is now at hand. It paves the way toward understanding of more involved phononic systems, for example, exhibiting confined phonon modes $[14,33]$ or phonon state preparation [6]. Funding. TK, DW \& PM acknowledge support form the Polish National Agency for Academic Exchange under the International Academic Partnerships program, the Würzburg group by the State of Bavaria and C.S by the DFG (project Schn1376-5.1). Disclosures. The authors declare no conflicts of interest.

\section{REFERENCES}

1. D. E. Reiter, T. Kuhn, M. Glässl, and V. M. Axt, J. Phys. Condens. Matter 26, 423203 (2014).

2. J. Huneke, A. Krügel, T. Kuhn, A. Vagov, and V. M. Axt, Phys. Rev. B 78, 085316 (2008).

3. M.-A. Lemonde, S. Meesala, A. Sipahigil, M. J. A. Schuetz, M. D. Lukin, M. Loncar, and P. Rabl, Phys. Rev. Lett. 120, 213603 (2018).

4. D. E. Reiter, D. Wigger, V. M. Axt, and T. Kuhn, Phys. Rev. B 84, 195327 (2011).

5. I. Wilson-Rae, P. Zoller, and A. Imamoğlu, Phys. Rev. Lett. 92, 075507 (2004).

6. A. Auffeves and M. Richard, Phys. Rev. A 90, 023818 (2014).

7. T. Hahn, D. Groll, T. Kuhn, and D. Wigger, Phys. Rev. B 100, 024306 (2019).

8. H. Fröhlich, Adv. Phys. 3, 325 (1954).

9. R. P. Feynman, Phys. Rev. 97, 660 (1955).

10. D. Wigger, S. Lüker, D. Reiter, V. M. Axt, P. Machnikowski, and T. Kuhn, J. Physics: Condens. Matter 26, 355802 (2014).

11. L. Besombes, K. Kheng, L. Marsal, and H. Mariette, Phys. Rev. B 63, 155307 (2001).

12. E. Peter, J. Hours, P. Senellart, A. Vasanelli, A. Cavanna, J. Bloch, and J. M. Gérard, Phys. Rev. B 69, 041307 (2004).

13. P. Palinginis, H. Wang, S. V. Goupalov, D. S. Citrin, M. Dobrowolska, and J. K. Furdyna, Phys. Rev. B 70, 073302 (2004).

14. G. Lindwall, A. Wacker, C. Weber, and A. Knorr, Phys. Rev. Lett. 99, 087401 (2007).

15. S. Kumar, M. Brotóns-Gisbert, R. Al-Khuzheyri, A. Branny, G. Ballesteros-Garcia, J. F. Sánchez-Royo, and B. D. Gerardot, Optica. 3, 882 (2016).

16. D. Wigger, R. Schmidt, O. Del Pozo-Zamudio, J. A. Preuß, P. Tonndorf, R. Schneider, P. Steeger, J. Kern, Y. Khodaei, J. Sperling, S. Michaelis de Vasconcellos, R. Bratschitsch, and T. Kuhn, 2D Mater. 6, 035006 (2019).

17. T. Jakubczyk, V. Delmonte, S. Fischbach, D. Wigger, D. E. Reiter, Q. Mermillod, P. Schnauber, A. Kaganskiy, J.-H. Schulze, A. Strittmatter, S. Rodt, W. Langbein, T. Kuhn, S. Reitzenstein, and J. Kasprzak, ACS Photonics 3, 2461 (2016).

18. G. M. Vanacore, J. Hu, W. Liang, S. Bietti, S. Sanguinetti, F. Carbone, and A. H. Zewail, Struct. Dynam. 4, 044034 (2017).

19. A. Vagov, V. M. Axt, T. Kuhn, W. Langbein, P. Borri, and U. Woggon, Phys. Rev. B 70, 201305 (2004).

20. P. Borri, W. Langbein, U. Woggon, V. Stavarache, D. Reuter, and A. D. Wieck, Phys. Rev. B 71, 115328 (2005).

21. W. Langbein and B. Patton, Opt. Lett. 31, 1151 (2006).

22. S. Maier, P. Gold, A. Forchel, N. Gregersen, J. Mørk, S. Höfling, C. Schneider, and M. Kamp, Opt. Express 22, 8136 (2014).

23. F. Fras, Q. Mermillod, G. Nogues, C. Hoarau, C. Schneider, M. Kamp, S. Höfling, W. Langbein, and J. Kasprzak, Nat. Photonics 10, 155 (2016).

24. A. Vagov, V. M. Axt, and T. Kuhn, Phys. Rev. B 67, 115338 (2003).

25. S. Lüker, T. Kuhn, and D. E. Reiter, Phys. Rev. B 96, 245306 (2017).

26. D. Wigger, D. E. Reiter, V. M. Axt, and T. Kuhn, Phys. Rev. B 87, 085301 (2013).

27. A. Krügel, A. Vagov, V. M. Axt, and T. Kuhn, Phys. Rev. B 76, 195302 (2007).

28. P. Borri, W. Langbein, S. Schneider, U. Woggon, R. L. Sellin, D. Ouyang, and D. Bimberg, Phys. Rev. B 66, 081306 (2002).

29. T. Braun, S. Betzold, N. Lundt, M. Kamp, S. Höfling, and C. Schneider, Phys. Rev. B 93, 155307 (2016).

30. D. Wigger, Q. Mermillod, T. Jakubczyk, F. Fras, S. Le-Denmat, D. E. Reiter, S. Höfling, M. Kamp, G. Nogues, C. Schneider, T. Kuhn, and J. Kasprzak, Phys. Rev. B 96, 165311 (2017).

31. D. Wigger, C. Schneider, S. Gerhardt, M. Kamp, S. Höfling, T. Kuhn, and J. Kasprzak, Optica 5, 1442 (2018).

32. B. Krummheuer, V. M. Axt, and T. Kuhn, Phys. Rev. B 65, 195313 (2002).

33. B. Krummheuer, V. M. Axt, and T. Kuhn, Phys. Rev. B 72, 245336 (2005). 


\section{FULL REFERENCES}

1. D. E. Reiter, T. Kuhn, M. Glässl, and V. M. Axt, "The role of phonons for exciton and biexciton generation in an optically driven quantum dot," J. Phys. Condens. Matter 26, 423203 (2014).

2. J. Huneke, A. Krügel, T. Kuhn, A. Vagov, and V. M. Axt, "Impact of strain waves traveling across a quantum dot on the optical response of the dot: Distinction between strain waves of different origin," Phys. Rev. B 78, 085316 (2008).

3. M.-A. Lemonde, S. Meesala, A. Sipahigil, M. J. A. Schuetz, M. D. Lukin, M. Loncar, and P. Rabl, "Phonon networks with silicon-vacancy centers in diamond waveguides," Phys. Rev. Lett. 120, 213603 (2018).

4. D. E. Reiter, D. Wigger, V. M. Axt, and T. Kuhn, "Generation and dynamics of phononic cat states after optical excitation of a quantum dot," Phys. Rev. B 84, 195327 (2011).

5. I. Wilson-Rae, P. Zoller, and A. Imamoğlu, "Laser cooling of a nanomechanical resonator mode to its quantum ground state," Phys. Rev. Lett. 92, 075507 (2004).

6. A. Auffeves and M. Richard, "Optical driving of macroscopic mechanical motion by a single two-level system," Phys. Rev. A 90, 023818 (2014).

7. T. Hahn, D. Groll, T. Kuhn, and D. Wigger, "Influence of excited state decay and dephasing on phonon quantum state preparation," Phys. Rev. B 100, 024306 (2019).

8. H. Fröhlich, "Electrons in lattice fields," Adv. Phys. 3, 325-361 (1954).

9. R. P. Feynman, "Slow electrons in a polar crystal," Phys. Rev. 97, 660 (1955).

10. D. Wigger, S. Lüker, D. Reiter, V. M. Axt, P. Machnikowski, and T. Kuhn, "Energy transport and coherence properties of acoustic phonons generated by optical excitation of a quantum dot," J. Physics: Condens. Matter 26, 355802 (2014).

11. L. Besombes, K. Kheng, L. Marsal, and H. Mariette, "Acoustic phonon broadening mechanism in single quantum dot emission," Phys. Rev. B 63, 155307 (2001).

12. E. Peter, J. Hours, P. Senellart, A. Vasanelli, A. Cavanna, J. Bloch, and J. M. Gérard, "Phonon sidebands in exciton and biexciton emission from single GaAs quantum dots," Phys. Rev. B 69, 041307 (2004).

13. P. Palinginis, H. Wang, S. V. Goupalov, D. S. Citrin, M. Dobrowolska, and J. K. Furdyna, "Exciton dephasing in self-assembled CdSe quantum dots," Phys. Rev. B 70, 073302 (2004).

14. G. Lindwall, A. Wacker, C. Weber, and A. Knorr, "Zero-phonon linewidth and phonon satellites in the optical absorption of nanowire-based quantum dots," Phys. Rev. Lett. 99, 087401 (2007).

15. S. Kumar, M. Brotóns-Gisbert, R. Al-Khuzheyri, A. Branny, G. Ballesteros-Garcia, J. F. Sánchez-Royo, and B. D. Gerardot, "Resonant laser spectroscopy of localized excitons in monolayer $\mathrm{WSe}_{2}$," Optica. 3, 882-886 (2016).

16. D. Wigger, R. Schmidt, O. Del Pozo-Zamudio, J. A. Preuß, P. Tonndorf, R. Schneider, P. Steeger, J. Kern, Y. Khodaei, J. Sperling, $\mathrm{S}$. Michaelis de Vasconcellos, R. Bratschitsch, and T. Kuhn, "Phononassisted emission and absorption of individual color centers in hexagonal boron nitride," 2D Mater. 6, 035006 (2019).

17. T. Jakubczyk, V. Delmonte, S. Fischbach, D. Wigger, D. E. Reiter, Q. Mermillod, P. Schnauber, A. Kaganskiy, J.-H. Schulze, A. Strittmatter, S. Rodt, W. Langbein, T. Kuhn, S. Reitzenstein, and J. Kasprzak, "Impact of phonons on dephasing of individual excitons in deterministic quantum dot microlenses," ACS Photonics 3, 2461-2466 (2016).

18. G. M. Vanacore, J. Hu, W. Liang, S. Bietti, S. Sanguinetti, F. Carbone, and A. H. Zewail, "Ultrafast atomic-scale visualization of acoustic phonons generated by optically excited quantum dots," Struct. Dynam. 4, 044034 (2017)

19. A. Vagov, V. M. Axt, T. Kuhn, W. Langbein, P. Borri, and U. Woggon, "Nonmonotonous temperature dependence of the initial decoherence in quantum dots," Phys. Rev. B 70, 201305 (2004).

20. P. Borri, W. Langbein, U. Woggon, V. Stavarache, D. Reuter, and A. D. Wieck, "Exciton dephasing via phonon interactions in InAs quantum dots: Dependence on quantum confinement," Phys. Rev. B 71, 115328 (2005).

21. W. Langbein and B. Patton, "Heterodyne spectral interferometry for multidimensional nonlinear spectroscopy of individual quantum systems,"
Opt. Lett. 31, 1151-1153 (2006).

22. S. Maier, P. Gold, A. Forchel, N. Gregersen, J. Mørk, S. Höfling, C. Schneider, and M. Kamp, "Bright single photon source based on selfaligned quantum dot-cavity systems," Opt. Express 22, 8136-8142 (2014).

23. F. Fras, Q. Mermillod, G. Nogues, C. Hoarau, C. Schneider, M. Kamp, S. Höfling, W. Langbein, and J. Kasprzak, "Multi-wave coherent control of a solid-state single emitter," Nat. Photonics 10, 155 (2016).

24. A. Vagov, V. M. Axt, and T. Kuhn, "Impact of pure dephasing on the nonlinear optical response of single quantum dots and dot ensembles," Phys. Rev. B 67, 115338 (2003).

25. S. Lüker, T. Kuhn, and D. E. Reiter, "Phonon impact on optical control schemes of quantum dots: role of quantum dot geometry and symmetry," Phys. Rev. B 96, 245306 (2017).

26. D. Wigger, D. E. Reiter, V. M. Axt, and T. Kuhn, "Fluctuation properties of acoustic phonons generated by ultrafast optical excitation of a quantum dot," Phys. Rev. B 87, 085301 (2013).

27. A. Krügel, A. Vagov, V. M. Axt, and T. Kuhn, "Monitoring the buildup of the quantum dot polaron: Pump-probe and four-wave mixing spectra from excitons and biexcitons in semiconductor quantum dots," Phys. Rev. B 76, 195302 (2007).

28. P. Borri, W. Langbein, S. Schneider, U. Woggon, R. L. Sellin, D. Ouyang, and D. Bimberg, "Rabi oscillations in the excitonic ground-state transition of InGaAs quantum dots," Phys. Rev. B 66, 081306 (2002).

29. T. Braun, S. Betzold, N. Lundt, M. Kamp, S. Höfling, and C. Schneider, "Impact of ex situ rapid thermal annealing on magneto-optical properties and oscillator strength of In (Ga) As quantum dots," Phys. Rev. B 93, 155307 (2016)

30. D. Wigger, Q. Mermillod, T. Jakubczyk, F. Fras, S. Le-Denmat, D. E. Reiter, S. Höfling, M. Kamp, G. Nogues, C. Schneider, T. Kuhn, and J. Kasprzak, "Exploring coherence of individual excitons in inas quantum dots embedded in natural photonic defects: influence of the excitation intensity," Phys. Rev. B 96, 165311 (2017).

31. D. Wigger, C. Schneider, S. Gerhardt, M. Kamp, S. Höfling, T. Kuhn, and J. Kasprzak, "Rabi oscillations of a quantum dot exciton coupled to acoustic phonons: coherence and population readout," Optica $\mathbf{5}$, 1442-1450 (2018)

32. B. Krummheuer, V. M. Axt, and T. Kuhn, "Theory of pure dephasing and the resulting absorption line shape in semiconductor quantum dots," Phys. Rev. B 65, 195313 (2002).

33. B. Krummheuer, V. M. Axt, and T. Kuhn, "Coupled polarization and acoustic-phonon dynamics after optical excitation of quantum dots near surfaces," Phys. Rev. B 72, 245336 (2005). 